\title{
Comparison of phoropter and trial frame-based von Graefe heterophoria measures in non-presbyopic participants
}

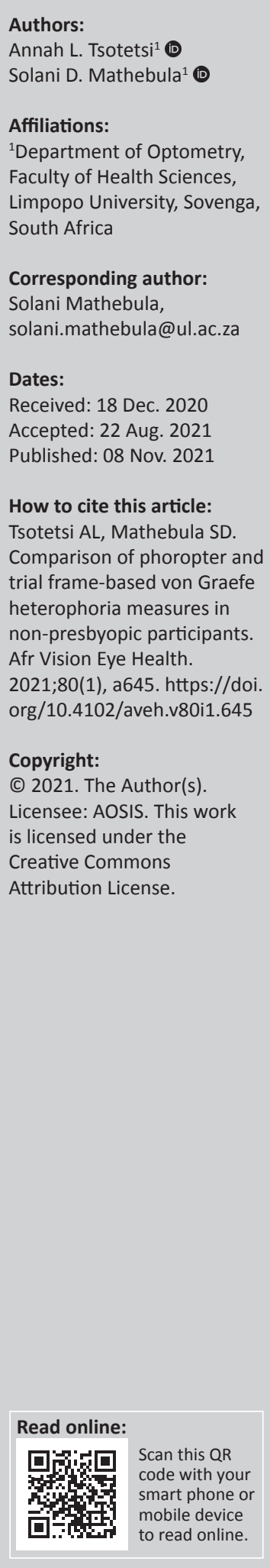

Background: There are several clinical techniques for the subjective measurement of heterophoria. In South Africa, von Graefe (VG) is a commonly used technique to quantify heterophoria using the phoropter.

Purpose: The purpose of the study was to investigate the agreement of VG heterophoria measurement using the phoropter and a trial frame.

Setting: The study was conducted at an Optometry Clinic, University of Limpopo, South Africa.

Methods: Both distance and near horizontal and vertical heterophoria VG measurements were performed on 88 visually-normal university students using the phoropter and trial frame. The handheld rotary prism was used on the trial frame. The $95 \%$ limits of agreement in prism diopters were compared using the Bland-Altman statistical test.

Results: For distance horizontal heterophoria, the VG means were 0.39 exophoria $\pm 2.0^{\Delta}$ and 0.38 exophoria $\pm 1.8^{\Delta}$ with the phoropter and trial frame respectively. The means for near horizontal heterophoria were 3.69 exophoria $\pm 3.3^{\Delta}$ and 4.13 exophoria $\pm 3.27^{\Delta}$ with the phoropter and trial frame. For the vertical heterophoria at distance, the means and standard deviations were 0.18 hyperphoria \pm 0.74 and 0.13 hyperphoria $\pm 0.07^{\star}$, while at near vision they were 0.03 hyperphoria \pm 0.71 and 0.07 hyperphoria $\pm 0.71^{\triangle}$, respectively.

Conclusion: Measurement of VG heterophoria testing using the phoropter and trial frame showed a high level of agreement for both distance and near vision performed through the phoropter and a trial frame. For clinical and research purposes, the phoropter and trial frame can be used interchangeably for measuring heterophoria.

Keywords: heterophoria; phoropter; trial frame; von Graefe; prism.

\section{Introduction}

Binocular vision is a unitary process which pertains to the bilateral motor coordination of the two eyes of an individual, and the subsequent sensory components that unifies their respective views of the world. ${ }^{1}$ The motor component positions and aligns the eyes in such a manner that sensory fusion can be maintained. However, if the motor and sensory fusion components of binocular vision are artificially suspended, a relative deviation of the visual axes may occur, in most individuals, and this condition is called heterophoria (phoria) ${ }^{1,2}$ Heterophoria is the physiological position of rest that is assumed by the eyes when fusion is disrupted. ${ }^{2}$ Rosenfield ${ }^{3}$ defined heterophoria as the locus of intersection of the lines of sight or visual axes, measured with respect to the object of regard, in the absence of a fusional vergence response. The Dictionary of Visual Science defines heterophoria as the tendency of the lines of sight to deviate from the relative positions necessary to maintain single binocular vision for a given distance of fixation. ${ }^{4}$ Generally, heterophoria is the latent deviation of the visual axes that is elicited when the fusion is artificially suspended by excluding one eye from participating in binocular vision. ${ }^{1}$

Esophoria or exophoria occurs when the visual axes of the eyes converge or diverge when the eyes are dissociated, respectively. Orthophoria occurs when the visual axes meet at the object of regard when the eyes are dissociated. 5,6 Hyperphoria or hypophoria occurs when one of the visual axis is higher than the other. The heterophoria position is measured by the amount of the prism necessary to align both visual axes at the point of fixation, and the magnitude is expressed in prism dioptres ( ${ }^{\Delta}$ or PD). However, when the fusion mechanism of binocular vision does not function fully or properly, a manifest deviation of one eye is present which is called heterotropia or strabismus. ${ }^{4}$ 
Clinically, a significant amount of heterophoria may lead to symptoms such as headache, blurred vision, diplopia, visual fatigue, nausea, and vertigo. ${ }^{2,7}$, The measurement of heterophoria is routinely assessed in clinical optometric or ophthalmologic practice to determine the binocular visual

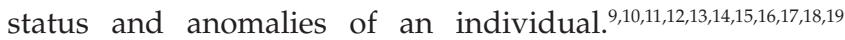
Diagnosis of conditions such as convergence insufficiency or convergence excess or divergence excess can be causes of the symptoms which can occur at distance, near or both. ${ }^{7}$ Accurate and repeatable clinical measurements of heterophoria are important when diagnosing and managing such conditions.

Several clinical methods have been established for the measurement of dissociated heterophoria, and many studies have been published on their reliability and regarding comparisons between different measurement methods. ${ }^{6,7,8,9,10,11,12,13,14,15,16,17,18,19}$ Most methods of heterophoria measurements are subjective. The von Graefe (VG) method is a popular and common method for measuring heterophoria where dissociation is achieved with the use of either horizontal or vertical prisms. $910,11,12,13,14,15,16,17$ Although the use of the cover test in clinical practice is also common, it has possible limitations such as the results depend on the experience of the clinician. ${ }^{2,7}$ Some authors have reported that the minimum detectable ocular deviation using the cover test is approximately $2^{\Delta} \cdot{ }^{20,21,22}$ The heterophoria measurement via the cover test depends largely on the skill of the examiner to detect any eye movement. However, several studies have found that the alternating cover test using prism neutralisation provides excellent repeatability, both within and between examiners and even when an examiner is relatively inexperienced, ${ }^{2}$ and therefore the method is an important one in practice. Another technique commonly used in clinical practice is the VG method, which typically is an in-phoropter measurement method ${ }^{4,5,6,7}$ used by many clinicians as it can be performed before and immediately after ophthalmic refraction with the spectacle correction in place. However, several studies found that the VG method is less repeatable compared to the cover test or to others such as the modified Thorington test. ${ }^{9,10,11}$

The use of trial frames in clinical settings to measure heterophoria is important, ${ }^{18}$ although the Maddox $\operatorname{rod}^{8}, \mathrm{VG}^{17}$ and Modified Thorington ${ }^{16}$ procedures are commonly carried out using the phoropter with the target positioned in the midline primary position. However, the use of the phoropter during heterophoria measurements may affect the vergence responses because of proximal vergence, head and eye position changes, and restrictions of the peripheral visual field. 2,12,13 It is unknown whether heterophoria results produced by trial frame measurements in free space are similar to that from the phoropter, or whether the two methods are interchangeable.

Currently, most South African eye care practices use phoropters rather than trial frames to carry out heterophoria measurements and other tests. However, using a trial frame gives practitioners the opportunity for direct observation of the eyes in a natural setting. Because people have different head and eye positions and some have restricted peripheral visual fields or profound refractive errors, the use of trial frames can be beneficial for measurement and clinical practice.

The main aim of the present study therefore was to compare in young adults the VG horizontal and vertical heterophoria measurements using both the phoropter and the trial frame in free space. Should the methods be comparable, this will also allow for early interventions of great benefit where optometrists or others conduct eye and visual screenings in remote areas.

\section{Methods}

A total of 88 participants of mean age $20.97 \pm 3.30$ years (range: 18-39 years) were recruited to participate in the study; of these 58 were females and 30 males. Each participant provided written consent, and the study followed the principles of the Helsinki Declaration on human subjects. All participants had corrected or uncompensated visual acuity of $6 / 6$ or better in each eye and near vision of $20 / 20$ (6/6 equivalent). None of the participants had any ocular pathology, heterotropia, amblyopia or history of eye surgery. The presence of heterophoria rather than heterotropia was confirmed with the unilateral cover test. All participants in this study presented with good stereopsis.

\section{Procedures}

The VG heterophorias were assessed for two viewing distances, namely $(6 \mathrm{~m})$ and near vision $(0.4 \mathrm{~m})$ using both the phoropter and trial frame. Each participant was evaluated for distance horizontal heterophoria, distance vertical heterophoria, near horizontal deviation and near vertical deviation. All VG heterophoria tests were performed by the principal investigator (A.L.T.). The accepted standard techniques were used to evaluate the VG measurements through the phoropter and trial frame. The distance heterophoria was measured at $6 \mathrm{~m}$ with the participant's habitual distance prescription and distance interpupillary distance placed into the phoropter and trial frame.

\section{Phoropter}

Risley prisms were introduced before the phoropter with $12^{\perp}$ base-in before the right eye and $6^{\Delta}$ base-up in front of the left eye. When testing at $6 \mathrm{~m}$, an isolated $6 / 6$ line of letters was projected as the target; whilst testing at $0.4 \mathrm{~m}$, a $20 / 20(6 / 6$ equivalent) line of letters was viewed. For all the measurements, participants were instructed to fixate the lower target (non-moving) and keep the letters clear. For horizontal measurements, the magnitude of the $12^{\Delta}$ base-in was reduced until the participant reported that the two images were vertically aligned, where one image was directly below the other. The amount of the horizontal prism that brought the diplopic images into vertical alignment was recorded as the horizontal heterophoria in PD. For vertical measurements, the magnitude of the $6^{\Delta}$ base-up in front of the left eye was reduced in one-dioptre-steps until each 
participant reported that the two images appeared to be aligned, side by side. The amount of the prism that brought the diplopic images into horizontal alignment was recorded as the vertical heterophoria.

\section{Trial frame}

Handheld rotary prisms and loose prisms were used with a loose $6^{\Delta}$ base-up placed in front of the left (dissociating) eye and the handheld rotary prism of $12^{\Delta}$ base-in (measuring) was held in front of the right eye against the trial frame to measure the horizontal heterophoria. The same distance and target used with the phoropter was used in similar manner with the trial frame where the $12^{\Delta}$ base-in was reduced until alignment was achieved. However, at near vision, the participant held the reduced Snellen chart at $0.4 \mathrm{~m}$ attached on the trial frame with a $40 \mathrm{~cm}$ string. The magnitude of the $12^{\Delta}$ base-in prism in front of the right eye was reduced until the diplopic images were in vertical alignment.

For vertical heterophorias, a loose $12^{\Delta}$ prism base-in was used as a dissociating prism in front of the right eye. The handheld rotary prism of $6^{\Delta}$ base-up was held against the trial frame in front of the left eye. The $6^{\Delta}$ base-up was reduced until the diplopic images were horizontally aligned. As far as possible, the same VG procedure was used as for the phoropter.

\section{Statistical analysis}

Statistical analyses were performed using Statistical Package for Social Sciences (SPSS, Inc., Chicago, IL, USA) version 27. Tests of normality, skewness, kurtosis and histograms were used to decide whether samples for heterophoria measurements were normally distributed. The significance level was set at 0.05 and considered statistically significant if $p<0.05$. For the purposes of this study, exophoria and hyperphoria were represented with positive signs and esophoria and hypophoria with negative signs. Paired $t$-tests and Bland-Altman analysis were performed to determine the level of agreement of the VG heterophorias measured through a phoropter and trial frame, respectively. BlandAltman analysis was performed to determine the limits of agreement (LoA) between the phoropter and trial frame for distance and near measurements of horizontal and vertical heterophoria. The 95\% LoA are presented by the upper and lower lines (see Figure 7), which are equal to the mean difference (bias) \pm 1.96 standard deviations. The middle line is the mean difference. Based on the minimum detectable eye movement for the cover test, the maximum acceptable $95 \%$ limits were regarded as $\pm 2^{\Delta .}{ }^{20,21,22}$ Intraclass correlation coefficients (ICCs) were determined to assess reliability using a two-way mixed absolute agreement model.

\section{Results}

It is important to assess the normality of measurements because the normal distributed measurements are an underlying assumption of parametric testing. The software, SPSS runs the Kolmogorov-Smirnov (K-S) test and the
Shapiro-Wilk (S-W) test and produces an output of the data distribution (Table 1). The $S-W$ test is regarded as the best choice for testing normality because it provides a better statistical power compared to the K-S, which is commonly used to handle large samples of more than 50 participants. The measurements were somewhat skewed, but not markedly.

Table 2 shows the descriptive statistics for the VG heterophoria measurements using the phoropter and trial frame. Values for medians, skewness and kurtosis are also included. The mean heterophoria measurements for distance vision were $0.39^{\Delta} \pm 2.04^{\Delta}$ with a phoropter and $0.38^{\Delta} \pm 1.79^{\Delta}$ with a trial frame, $p>0.05$. The mean vertical heterophorias were $0.18^{\Delta} \pm 0.74^{\Delta}$ and $0.13^{\Delta} \pm 0.42^{\Delta}$ with the phoropter and trial frame, respectively, when $p>0.05$. The mean horizontal heterophoria at near vision with the phoropter was $3.69^{\Delta} \pm 3.25^{\Delta}$ and $4.13^{\Delta} \pm$ $3.27^{\Delta}$ with the trial frame, when $p>0.05$. Skewness and kurtosis are two important characteristics that describe the shape of a probability distribution. ${ }^{23}$ Skewness shows if the distribution is symmetric or not. Kurtosis of a normal univariate distribution is zero (0). A distribution with kurtosis less than 0 is platykurtic ${ }^{23}$ and produces fewer and less extreme outliers than does the normal distribution. The distribution with a kurtosis $>0$ is said to be leptokurtic and produces more outliers than the normal

TABLE 1: Tests of normality for Von Graefe heterophoria in 88 adults, aged 18-39 years, using the Kolmogorov-Smirnov and Shapiro-Wilk tests.

\begin{tabular}{|c|c|c|c|c|c|c|c|}
\hline \multirow[t]{2}{*}{ Methods } & \multirow[t]{2}{*}{ Distances } & \multicolumn{3}{|c|}{ Kolmogorov-Smirnov } & \multicolumn{3}{|c|}{ Shapiro-Wilk } \\
\hline & & Statistic & $d f$ & $p$ & Statistic & $d f$ & $p$ \\
\hline \multirow[t]{4}{*}{ Phoropter } & $\mathrm{H}_{\text {far }}$ & 0.2 & 87 & 0.07 & 0.8 & 87 & 0.08 \\
\hline & $\mathrm{H}_{\text {near }}$ & 0.4 & 87 & 0.09 & 0.9 & 87 & 0.09 \\
\hline & $V_{\text {far }}$ & 0.6 & 87 & 0.08 & 0.5 & 87 & 0.08 \\
\hline & $V_{\text {near }}$ & 0.3 & 87 & 0.08 & 0.6 & 87 & 0.06 \\
\hline \multirow[t]{4}{*}{ Trial frame } & $\mathrm{H}_{\text {far }}$ & 0.6 & 87 & 0.06 & 0.9 & 87 & 0.06 \\
\hline & $\mathrm{H}_{\text {near }}$ & 0.3 & 87 & 0.11 & 0.9 & 87 & 0.11 \\
\hline & $V_{\text {far }}$ & 0.7 & 87 & 0.09 & 0.6 & 87 & 0.70 \\
\hline & $V_{\text {near }}$ & 0.5 & 87 & 0.08 & 0.7 & 87 & 0.60 \\
\hline
\end{tabular}

Note: If $p>0.05$, samples are normally distributed and thus all samples were normally distributed here.

$d f$, degree of freedom.

$\mathrm{H}_{\text {far }}$ and $\mathrm{V}_{\mathrm{far}}$ represent horizontal and vertical heterophorias at $6 \mathrm{~m}$, whilst $\mathrm{H}_{\text {near }}$ and $\mathrm{V}_{\text {ner }}$ represent horizontal and vertical heterophorias at $0.4 \mathrm{~m}$.

TABLE 2: Descriptive statistics for the heterophoria measurements using the phoropter and trial frame.

\begin{tabular}{|c|c|c|c|c|c|c|c|c|}
\hline \multirow[t]{2}{*}{ Statistics } & \multicolumn{4}{|c|}{ Phoropter } & \multicolumn{4}{|c|}{ Trial frame } \\
\hline & $\mathbf{H}_{\mathrm{far}}$ & $\mathbf{H}_{\text {near }}$ & $\mathbf{V}_{\mathrm{far}}$ & $v_{\text {near }}$ & $\mathbf{H}_{\text {far }}$ & $\mathbf{H}_{\text {near }}$ & $\mathbf{V}_{\mathrm{far}}$ & $V_{\text {near }}$ \\
\hline Means & 0.39 & 3.69 & 0.18 & 0.03 & 0.38 & 4.13 & 0.13 & 0.07 \\
\hline s.d. & 2.04 & 3.25 & 0.74 & 0.71 & 1.79 & 3.27 & 0.42 & 0.71 \\
\hline Ranges & -6 to 8 & -5 to 15 & -1 to 1 & -3 to 1 & -4 to 6 & -4 to 15 & -1 to 1 & -3 to 2 \\
\hline Medians & 0.00 & 4.00 & 0.00 & 0.00 & 0.00 & 4.00 & 0.00 & 0.00 \\
\hline Skewness & 0.33 & 0.23 & 4.13 & -1.88 & 0.38 & -0.11 & 0.77 & -0.90 \\
\hline Kurtosis & 2.01 & 1.54 & 1.87 & 7.23 & 0.23 & 1.12 & 1.87 & 5.24 \\
\hline \multicolumn{9}{|l|}{ IQR } \\
\hline $\begin{array}{l}\text { 25th } \\
\text { Percentile }\end{array}$ & 0.00 & 0.00 & 0.00 & 0.00 & -1.00 & 2.00 & 0.00 & 0.00 \\
\hline $\begin{array}{l}\text { 50th } \\
\text { Percentile }\end{array}$ & 0.00 & 1.00 & 0.00 & 0.00 & 0.00 & 4.00 & 0.00 & 0.00 \\
\hline $\begin{array}{l}\text { 75th } \\
\text { Percentile }\end{array}$ & 2.00 & 6.00 & 0.00 & 0.00 & 2.00 & 6.00 & 0.00 & 0.00 \\
\hline
\end{tabular}

Note: Units are in prism dioptres $\left({ }^{\Delta}\right)$. Exophoria and hyperphoria were assigned positive whilst esophoria and hypophoria were given negative signs.

s.d., standard deviation; IQR, interquartile range.

$\mathrm{H}_{\text {far }}$ and $\mathrm{V}_{\text {far }}$ represent horizontal and vertical heterophorias at $6 \mathrm{~m}$, whilst $\mathrm{H}_{\text {near }}$ and $\mathrm{V}_{\text {near }}$ represent horizontal and vertical heterophorias at $0.4 \mathrm{~m}$. 
distribution (see Table 2). Most samples are leptokurtic although the horizontal heterophoria at far and near are almost zero and mesokurtic.

Figure 1 depicts the box plots for the horizontal heterophorias measured using the phoropter and trial frame. Each box plot shows the median as a horizontal bold line inside the box and the interquartile range (IQR) between the 25th and 75th percentiles as the length of the box. The lines extending from the top and bottom of the box are called the whiskers, and small lines at the ends of the whiskers represent values that are 1.5 times the IQR from either end of the box. Measurements greater than 1.5 times the IQR concerned are outliers represented by circles, whilst those represented by asterisks are extreme outliers and are either more or less 3 times the IQR concerned.

The distribution of horizontal and vertical heterophorias are presented in Figures 2-5. The histograms plot the VG heterophoria measurements against their frequencies. The

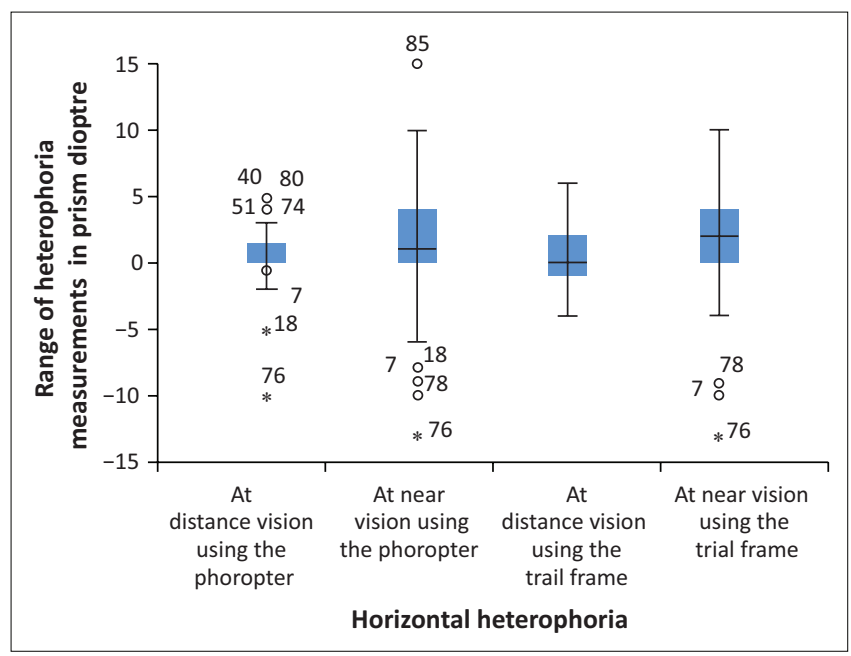

Note: The sample consists of 88 participants, aged $18-39$ years.

FIGURE 1: Box plots for the measurements of horizontal heterophorias using the phoropter and trial frame. histograms provide information about data normality, that is, whether the data match the curve (and normal bell shape) as indicated in the graphs.

The relationship between the VG heterophoria measures using the phoropter and trial frame was evaluated using the correlation coefficient $(r)$ between the results of the phoropter and trial frame (see Table 3 and Figure 6). The correlation showed how strongly related was the relationship of the heterophoria obtained using the phoropter and trial frame . This gives an idea of strength of the linear relationship and is only interpreted if $p<0.05$. Correlation quantifies the degree to which the two methods are related, however, a high correlation does not imply that there is good agreement between the two methods.

Table 4 shows the mean differences amongst the distance and near heterophoria measurements. The mean differences

TABLE 3: Pearson coefficients of correlations between heterophoria measured using the phoropter and trial frame.

\begin{tabular}{lcl}
\hline Paired heterophoria measures & Correlation $(r)$ & Sig. \\
\hline Horizontal heterophoria at far & 0.8 & 0.00 \\
Horizontal heterophoria at near & 0.8 & 0.00 \\
Vertical heterophoria at far & 0.9 & 0.00 \\
Vertical heterophoria at near & 0.9 & 0.00 \\
\hline
\end{tabular}

Note: All were significant correlated at $p<0.05$.

Sig., significance.

TABLE 4: The mean differences of heterophoria measurements and 95\% exact confidence intervals around the means.

\begin{tabular}{|c|c|c|c|c|}
\hline \multirow[t]{2}{*}{ Paired mean differences } & \multirow[t]{2}{*}{ Mean \pm s.d. } & \multicolumn{2}{|c|}{$5 \%$ confidence intervals } & \multirow[t]{2}{*}{ Sig. value } \\
\hline & & Lower & Upper & \\
\hline $\mathrm{H}_{\mathrm{far}}$ & $0.01 \pm 1.29$ & -0.262 & 0.285 & 0.934 \\
\hline $\mathrm{H}_{\text {near }}$ & $-0.432 \pm 2.022$ & -0.860 & -0.003 & 0.048 \\
\hline $\mathrm{V}_{\mathrm{far}}$ & $-0.057 \pm 0.613$ & -0.073 & 0.187 & 0.387 \\
\hline$v_{0}$ & $-0.034 \pm 0.322$ & -0.103 & 0.034 & 0.320 \\
\hline
\end{tabular}

Note: $\mathrm{H}_{\mathrm{far}}$ and $\mathrm{V}_{\mathrm{far}}$ are mean differences of horizontal and vertical distance heterophoria measures, whilst $H_{\text {near }}$ and $V_{\text {near }}$ are for horizontal and vertical heterophorias at near vision. s.d., standard deviation; Sig., significance

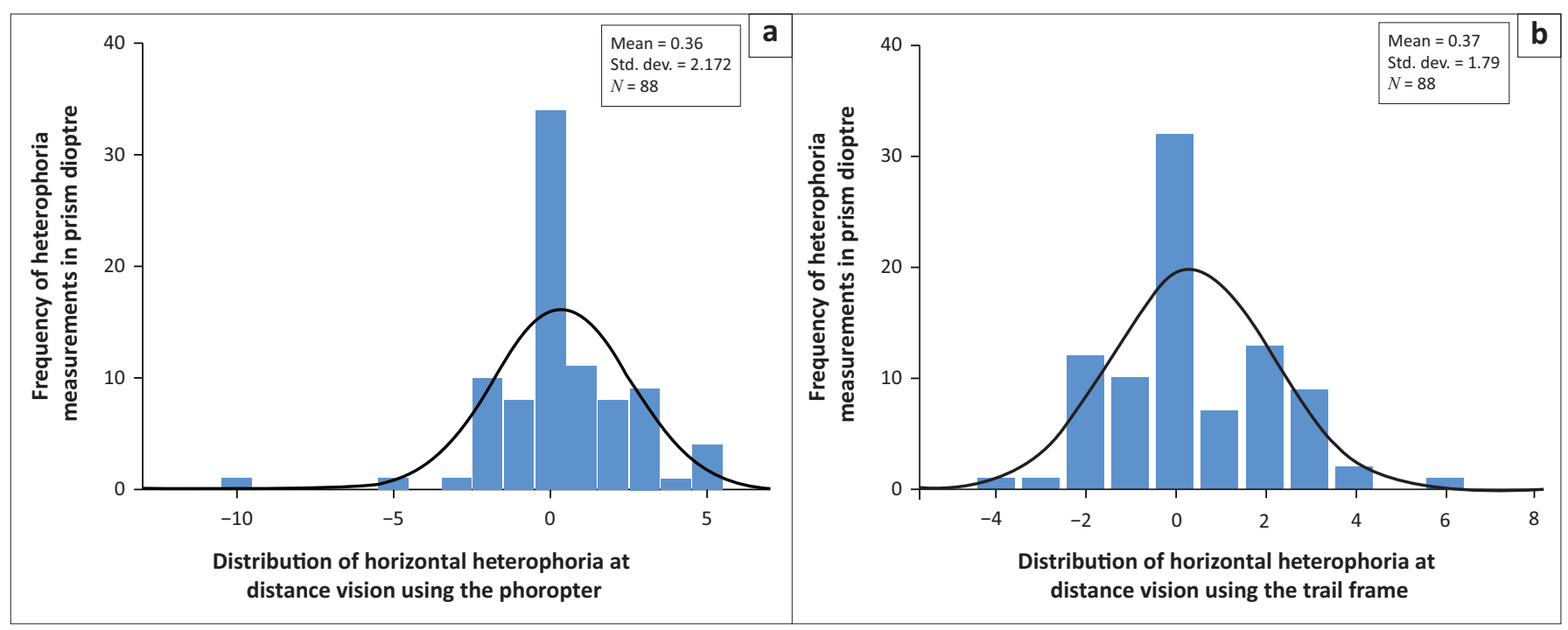

Note: Units are in prism dioptres.

Std. Dev., standard deviation.

FIGURE 2: Histograms for distance horizontal heterophoria using the phoropter (a) and trial frame (b) 


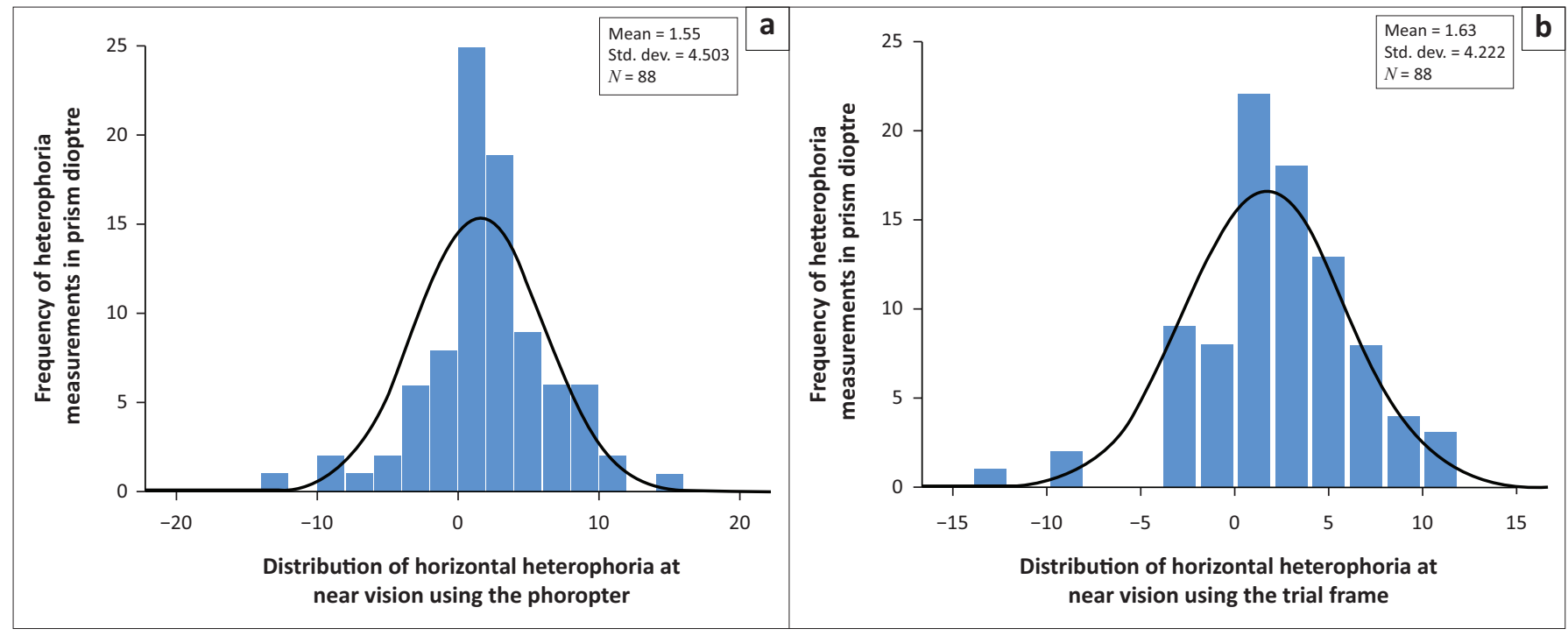

Note: Units are in prism dioptres.

Std. Dev., standard deviation.

FIGURE 3: Histograms for near horizontal heterophoria using the phoropter (a) and trial frame (b).

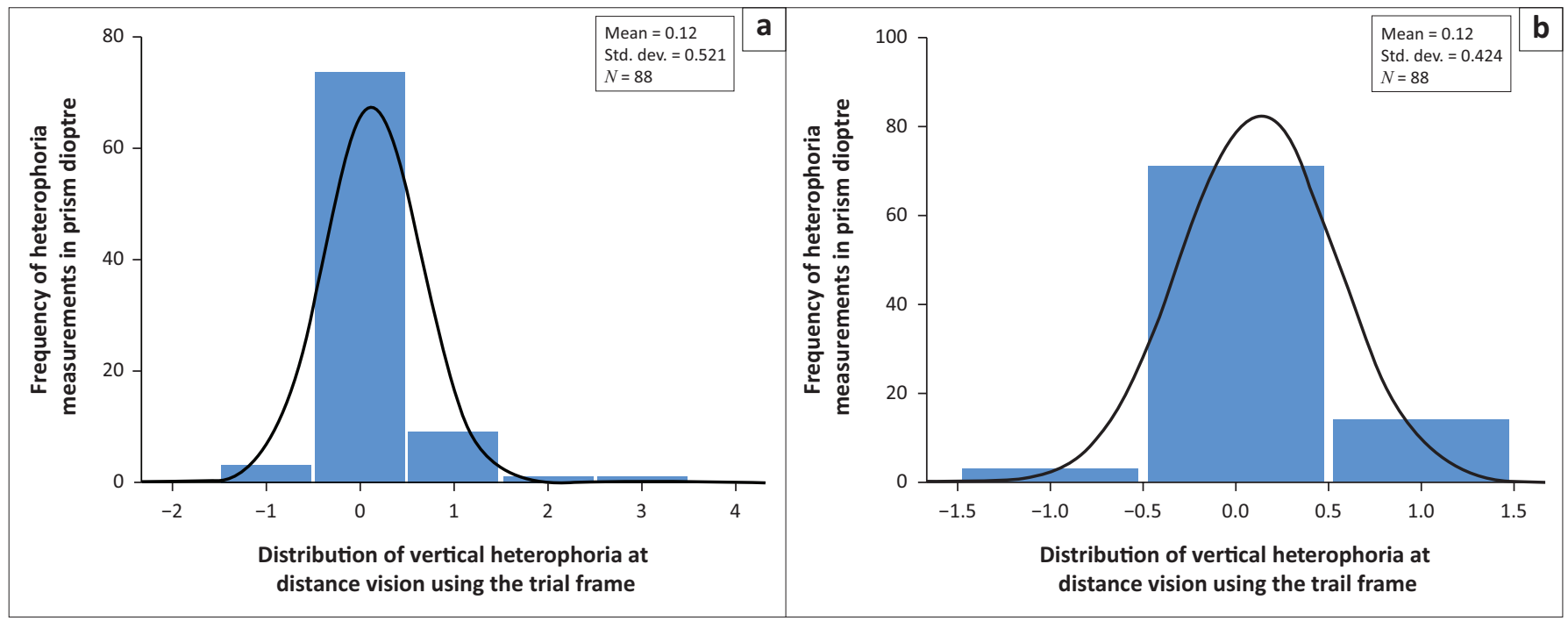

Note: Units are in prism dioptres.

Std. Dev., standard deviation.

FIGURE 4: Histograms for distance vertical heterophoria using the phoropter (a) and trial frame (b).

are indicated in Bland-Altman plots (Figures 7-10). The plots are based on the means of the two distance or near heterophoria measurements against their mean differences. The mean differences are represented by the red solid horizontal lines, whilst the $95 \%$ LoA are represented by the bold upper and lower lines. The upper and the lower LoA are equal to the mean difference (bias) $\pm 1.96 \times$ s.d. Confidence intervals (CI) $(5 \%)$ for the LoA are represented by the dotted lines.

Figures 7-10 show the Bland-Altman analysis for the heterophoria measurements using the phoropter and trial frame for distance and near vision. The Bland-Altman plots here show both the 95\% LoA (see Table 4) and also an interval for acceptable or good agreement, based on the minimum detectable ocular deviation using the cover test, that is, $\pm 2^{\Delta}$. The graphs show the mean differences (biases) per comparison and $95 \%$ of the differences between the phoropter and trial frame.

For distance horizontal heterophoria, the differences between the two methods range over a total interval of $4^{\Delta}$. The interval of horizontal heterophoria for near vision ranged over a total interval of $8^{\Delta}$. The Bland-Altman graphs showed an overall mean difference of less than $2^{\Delta}$ for all heterophoria measurements. However, the LoA for the horizontal heterophoria at distance vision fell outside the predetermined criterion of $\pm 2^{\Delta}$. The near horizontal heterophoria and all vertical heterophorias had LoA within $2^{\perp}$.

The ICC is very commonly used for reliability on quantitative data. ${ }^{24}$ Before any measurement instrument or tool can be used for clinical or research application, its 


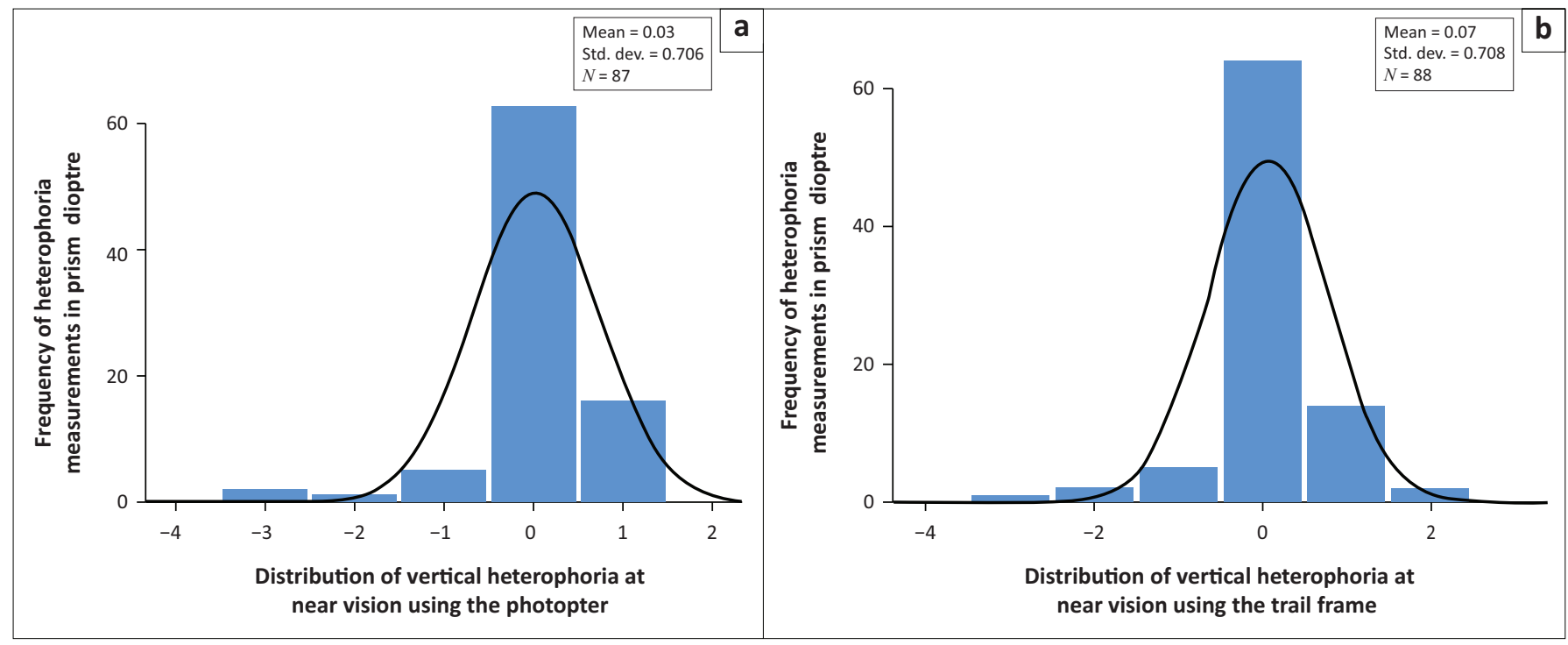

Note: Units are in prism dioptres.

Std. Dev., standard deviation.

FIGURE 5: Histograms for near vertical heterophoria using (a) the phoropter and (b) trial frame.

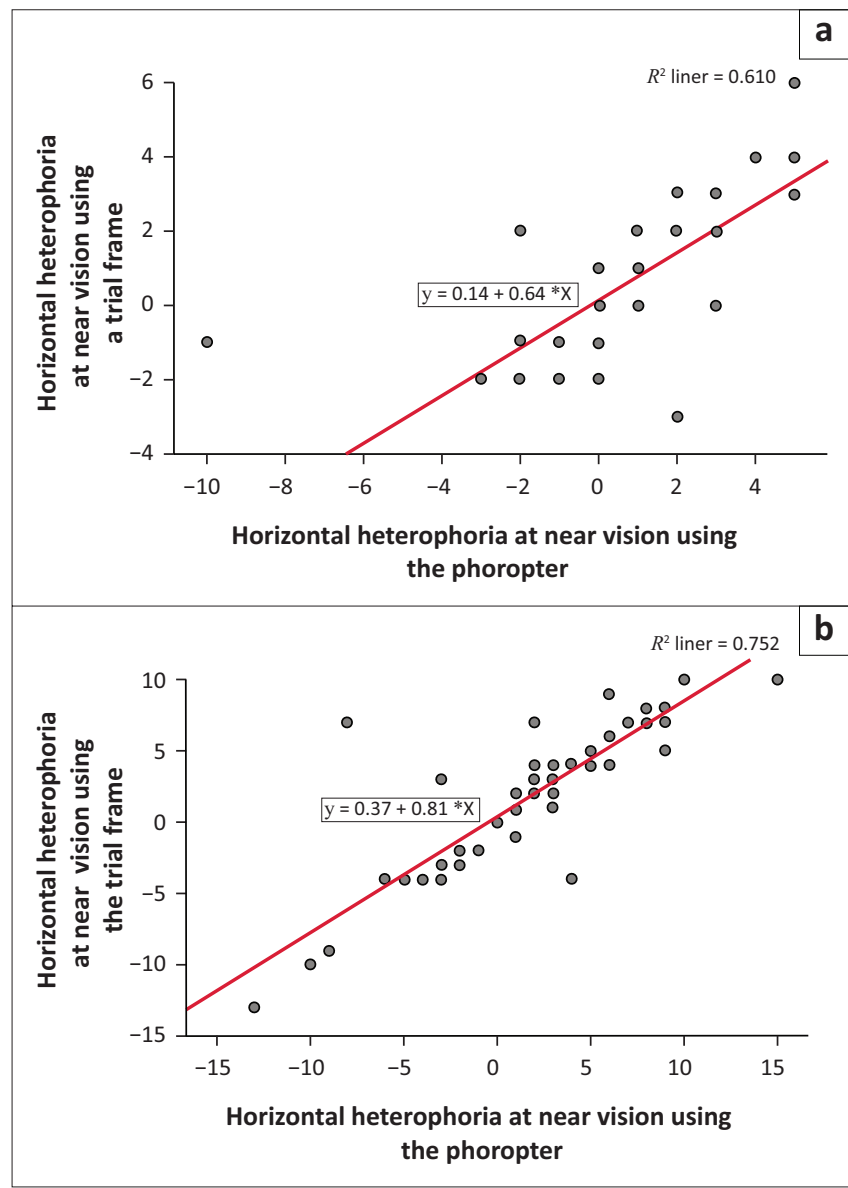

FIGURE 6: Scatter plots of horizontal heterophoria measurements at distance (a) and near vision (b).

reliability should be estimated. Reliability can be defined as an extent to which measurements can be replicated, and the ICC is the reliability index. Reliability value ranges between 0 and 1 , with values closer to 1 representing stronger reliability. The ICC values less than 0.5 are indicative of poor reliability, values between 0.5 and 0.75 indicate moderate reliability, values between 0.75 and 0.9 indicate

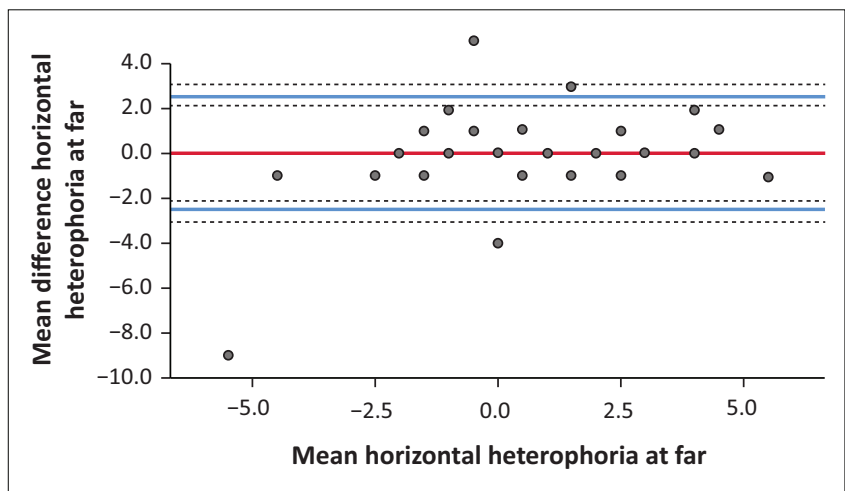

The red solid line represents the mean difference. The $95 \%$ limits of agreement are shown in blue solid lines, whilst the $95 \%$ confidence intervals about the limits are represented by dashed lines. Units are in prism dioptres. Thin grey lines at -2 and 2 represent acceptable or good agreement, that is, $\pm 2^{\Delta}$ about zero difference; most but not all differences were within $\pm 2^{\triangle}$ and the same applies to the $95 \%$ limits of agreement.

FIGURE 7: Bland-Altman plot showing the mean difference (red line) between the horizontal heterophoria measured for distance vision $(6 \mathrm{~m})$ using the phoropter and trial frame.

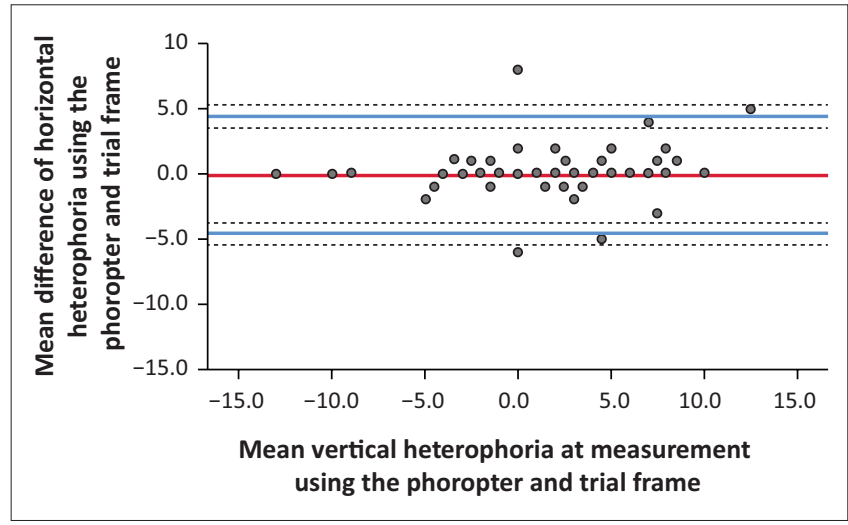

The $95 \%$ limits of agreement are shown with blue lines and the $95 \%$ confidence intervals around limits of agreement are indicated with dotted lines. Units are in prism dioptres.

FIGURE 8: Bland-Altman plot for the mean difference (red line) between the horizontal heterophoria measured at near vision $(0.4 \mathrm{~m})$ using the phoropter and trial frame.

good reliability and values greater than 0.9 indicate excellent reliability (see Table 5). 


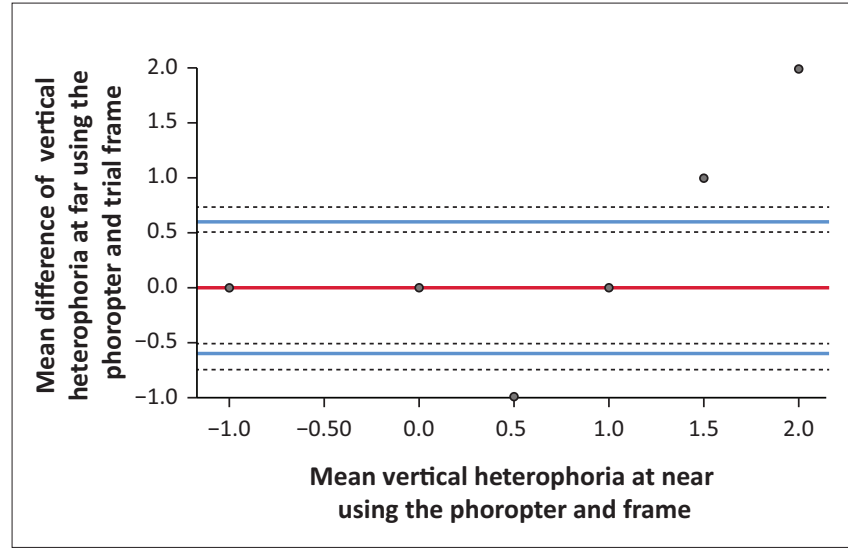

The $95 \%$ limits of agreement are shown with blue lines and $95 \% \mathrm{Cl}$ around limits of agreement are shown using dotted lines. Units are in prism dioptres.

FIGURE 9: Bland-Altman plot for mean difference (red line) between the vertical heterophoria measured at distance vision using the phoropter and trial frame.

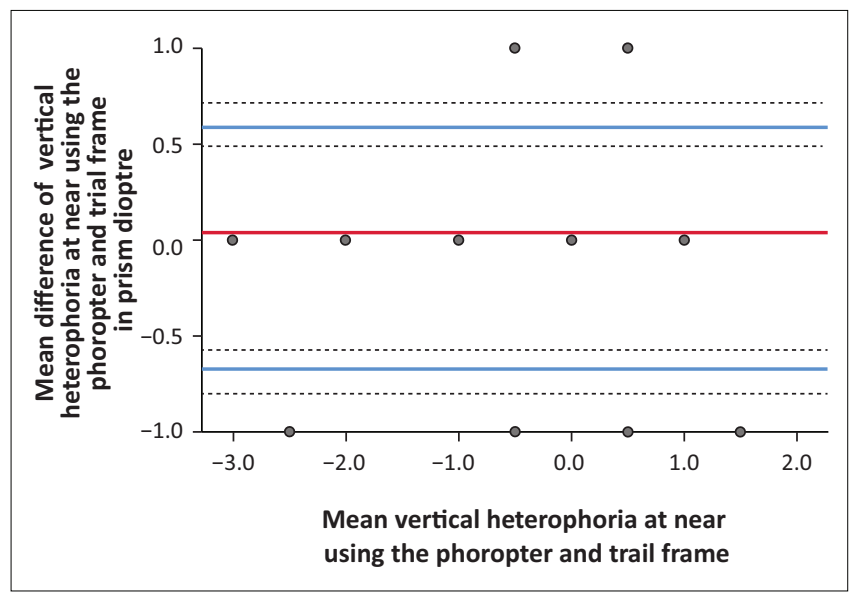

The $95 \%$ limits of agreement are shown with solid blue lines. Units are in prism dioptres. FIGURE 10: Bland-Altman graph showing the mean difference (red line) between the vertical heterophoria measured at near vision using the phoropter and trial frame.

TABLE 5: Intraclass correlation of heterophoria measurements using the phoropter and trial frame.

\begin{tabular}{|c|c|c|c|c|c|c|c|}
\hline \multirow[t]{2}{*}{ Items } & \multirow[t]{2}{*}{$\begin{array}{l}\text { Intraclass } \\
\text { correlation }\end{array}$} & \multicolumn{2}{|c|}{$\begin{array}{l}95 \% \text { confidence } \\
\text { interval }\end{array}$} & \multicolumn{4}{|c|}{$\begin{array}{l}F \text { Test with } \\
\text { true value } 0\end{array}$} \\
\hline & & Lower & Upper & Value & $d f 1$ & $d f 2$ & Sig. \\
\hline Single measure & 0.2 & 0.2 & 0.3 & 3.7 & 8.6 & 602 & 0.00 \\
\hline Average measure & 0.7 & 0.6 & 0.8 & 3.7 & 86 & 602 & 0.00 \\
\hline
\end{tabular}

Sig., significance; $d f 1=$ degree of freedom $1 ; d f 2=$ degree of freedom 2 .

Cronbach alpha also provides a measure of the internal consistency of a test. ${ }^{25}$ It is also expressed as a number between 0 and 1 . In this study, the Cronbach alpha is 0.7 . Acceptable values of alpha range from 0.70 to 0.95 .

\section{Discussion}

Measurements of heterophoria are a standard clinical procedure, and they are influential in the diagnosis and treatment plan of binocular vision. It is typically assessed by presenting non-fusible stimuli and determining the relative position of the two images. The heterophoria position is dependent on the level of the tonic convergence, accommodative position, proximal convergence, and vergence adaptation. ${ }^{1,2,3}$
This study investigated the agreement of VG heterophoria measurements using either a phoropter or trial frame. Two methods designed to measure the same variable should have high correlation. However, high correlation does not automatically indicate good agreement between the two methods of concern. Based on the defined limit of maximum acceptable differences of $2^{\Delta}$, the study showed that VG heterophoria measurements using the phoropter and trial frame are comparable and interchangeable in clinical settings.

Casillas and Rosenfield ${ }^{12}$ also compared subjective heterophoria testing with a phoropter and trial frame. Distance and near horizontal and vertical heterophorias were measured on 60 visually normal subjects between 20 and 34 years of age. The authors concluded that the use of a trial frame is more repeatable than when using a phoropter as measurement in free space provides more repeatable responses.

When the eyes view a distant object, the visual axes of the two eyes are parallel with little or no ocular accommodation. The majority of the 88 adult participants exhibited orthophoria when fixating at distance vision for both horizontal and vertical heterophoria, whether measured with a phoropter or a trial frame $(p>0.05)$. The range of distance horizontal heterophoria extended from $6^{\Delta}$ esophoria to $8^{\Delta}$ exophoria. The results of this study agree with findings of several authors who also found orthophoria at distance vision. ${ }^{5,8,18}$ This shows that there is a high prevalence of distance orthophoria despite many mechanical, neural and sensory variables. ${ }^{18,26,27}$

When looking at near objects, the eyes assume the active or functional position. ${ }^{2}$ According to the Maddox classification, the fusional convergence brings the eyes from fusion-free or primary position to the active position. ${ }^{1,2}$ The convergent position assumed by the eyes at near relative to distance position is proximal convergence and accommodative convergence. ${ }^{2}$ The proximal convergence is induced by the knowledge that the object of regard is located nearer to the observer, whilst accommodative convergence is stimulated by the consensual linkage between accommodation and convergence. Therefore, accommodation and convergence determine the position of the visual axes whilst looking at near objects.

The means for the near horizontal heterophorias were $3.69^{\Delta}$ exophoria with the phoropter and $4.13^{\Delta}$ exophoria with the trial frame test. The mean difference was $0.4^{\Delta} \pm 2^{\Delta}$ esophoria with the phoropter than the trial frame (see Table 4). However, there was a strong correlation $(r=0.8)$ between the near horizontal heterophorias measured using the phoropter and trial frame (see Figure 6b). Several studies have also found that the average near horizontal heterophoria is $3-6^{\Delta}$ exophoria. $6,7,8,9,10,11,12,13,14,15,16,17,18$

A vertical heterophoria occurs when the covered eye drifts up or down. Not much is known about vertical heterophoria, 
but several papers reported that the prevalence of vertical heterophoria in asymptomatic individuals ranges from $1-2^{\Delta .8,18}$ Vertical heterophoria is associated with symptoms of motion sickness, dizziness and nausea, ${ }^{28,29,30,31,32}$ and may also cause symptoms of double vision, head tilt and eyestrain. ${ }^{27}$ Albeit in a small sample, this study showed that vertical heterophorias are seldom more than $1^{\Delta}$ and rarely more than $2^{\Delta}$. The vertical heterophorias, both distance and near showed similar results, meaning they are less likely to change between distance and near vision. ${ }^{2,18,19}$ Small vertical heterophoria can give more severe symptoms than same magnitude horizontal heterophoria as the vertical fusional reserves are very small, rarely more than $4^{\Delta}$ in comparison with lateral reserves. ${ }^{28,29,30,31,32}$

Limitations of this study included the use of a single examiner for all the heterophoria techniques. The examiner was aware of the results of previous tests, but possible biases were perhaps reduced by the use of the standard procedures for heterophoria measures. The samples were relatively small and habitual prescriptions were used rather than current subjective refraction or best determined compensations. Measurements were also done on asymptomatic participants who were able to respond properly.

\section{Conclusion}

The findings of this study showed that the use of either the phoropter or trial frame provides a high level of agreement for both distance and near vision heterophoria measurements. Thus, patients with symptoms of uncompensated heterophoria at distance and or near vision can be screened using the trial frame in locations where there is no phoropter available. They (phoropter or trial frame) can also be used interchangeably in clinical settings, however, the use of trial frame can be slightly more challenging in setting up the use of prisms for the measurement of heterophoria.

\section{Acknowledgements}

The authors thank all those who participated in the study.

\section{Competing interests}

The authors declare that they have no financial or personal relationships that may have inappropriately influenced them in writing this article.

\section{Authors' contributions}

Both the authors contributed equally to the work. A.L.T. collected data and S.D.M. analysed data, and both were involved in the writing of the manuscript.

\section{Ethical considerations}

Ethical approval to conduct the study was received from the University of Limpopo, Turfloop Research Ethics Committee (REC-0310111-031, TREC/90/2019:PG).

\section{Funding information}

This research received no specific grant from any funding agency in the public, commercial or not-for-profit sectors.

\section{Data availability}

Data sharing is not applicable to this article.

\section{Disclaimer}

The views and opinions expressed in this article are those of the authors and do not necessarily reflect the official policy or position of any affiliated agency of the authors.

\section{References}

1. Griffin JR, Grisham JD. Binocular anomalies: Diagnosis and vision therapy. 3rd ed. London: Butterworth-Heinemann; 1995.

2. Rabbetts RB. Bennett and Rabbetts' clinical visual optics. 4th ed. London: Butterworth-Heinemann; 2007.

3. Rosenfield M. Tonic vergence and vergence adaptation. Opt Vis Sci. 1997;74(5):303-328. https://doi.org/10.1097/00006324-199705000-00027

4. Hofstetter HW, Griffin JR, Berman MS. Dictionary of visual science and related clinical term. 5th ed. Boston, MA: Buttersworth-Heinemann; 2000.

5. Dowley D. Heterophoria. Opt Vis Sci. 1990;67(6):456-460. https://doi. org/10.1097/00006324-199006000-00010

6. Walline JJ, Mutti DO, Zadnik K. Development of phoria in children. Optom Vis Sci. 1998;75(8):605-610. https://doi.org/10.1097/00006324-199808000-00026

7. Maples WC, Savoy RS, Harville BJ. Comparison of distance and near heterophoria by two clinical methods. Optom Vis Dev. 2009;40(2):100-106.

8. Letourneau JE, Giroux R. Nongaussian distribution curve of heterophorias among children. Opt Vis Sci. 1991;68(2):132-137. https://doi.org/10.1097/00006324199102000-00008

9. Goss DA, Reynolds JL, Todd RE. Comparison of four dissociated phoria tests: Reliability, and correlation with symptom survey scale. J Behav Optom. 2010;21(4):99-104.

10. Rainey BB, Schroeder TL, Goss DA. Inter-examiner repeatability of heterophoria tests. Opt Vis Sci. 1998;75(10):719-726. https://doi.org/10.1097/00006324199810000-00016

11. Schroeder TL, Rainey BB, Goss DA. Reliability of and comparison among methods of measuring dissociated phoria. Opt Vis Sci. 1996;73(6):389-397. https://doi. org/10.1097/00006324-199606000-00006

12. Cassilas EC, Rosenfield M. Comparison of subjective heterophoria testing with a phoropter and trial frame. Optom Vis Sci. 2006;83(4):237-241. https://doi. org/10.1097/01.opx.0000214316.50270.24

13. Sanker N, Prabhu A, Ray A. A comparison of near-dissociated heterophoria test in free space. Clin Exp Optom. 2012;95(11):638-642. https://doi.org/10.1111/ j.1444-0938.2012.00785.x

14. Canto-Cerdan M, Cucho-Martinez P, Garcia-Munoz A. Measuring the heterophoria: Agreement between two methods in non-presbyopic and presbyopic patients. J Optom. 2018;11(3):153-159. https://doi.org/10.1016/j. optom.2017.10.002

15. Anstice NS, Davidson B, Field B, Mathan J, Collins, AV, Black, JM. The repeatability and reproducibility of four techniques for measuring horizontal heterophoria: Implications for clinical practice. J Optom. 2021;14(3):275-281. https://doi org/10.1016/j.optom.2020.05.005

16. Goss DA, Moyer BJ, Teske MC. A comparison of dissociated phoria test findings with von Graefe phorometry and modified Thorington testing. J Behav Optom. 2008;19(6):145-149.

17. Jackson TW, Goss DA. Variation and correlation of standard clinical phoropter tests of phorias, vergence ranges, and relative accommodation in a sample of school-age children. J Am Optom Assoc. 1991;62(7):540-547.

18. Mathebula SD, Sheni DDD, Oduntan AO. Distribution of heterophoria among primary school children of South Africa. S Afr Optom. 2002;61(2):48-54.

19. Makgaba NT. A retrospective analysis of heterophoria values in a clinical population aged 18 to 30 years. Afr Vision Eye Health. 2006;65(4):150-156. https://doi.org/10.4102/aveh.v65i4.270

20. Fogt N, Baughman BJ, Good G. The effect of experience on the detection of small eye movements. Opt Vis Sci. 2000;77(12):670-674. https://doi.org/10.1097/00006324200012000-00014

21. Romano DE, Noorden GK. Limitations of cover test in detecting strabismus. Am J Ophthalmol. 1971;72(1):10-12. https://doi.org/10.1016/0002-9394(71)91585-6

22. Ludvigh $\mathrm{E}$. Amount of eye movement objectively perceptible to the unaided eye. Am J Ophthalmol. 1949;32(5):649-650. https://doi.org/10.1016/00029394(49)91415-4 
23. Sharma R, Bhandari RK. Skewness, kurtosis and Newton's inequality. Rocky Mountain J Math. 2015;45(5):1639-1643. https://doi.org/10.1216/RMJ-2015-455-1639

24. Terry KK, Mae YL. A guideline of selecting and reporting intraclass correlation coefficient for reliability research. J Chiropract Med. 2016;15(2):155-163. https:// doi.org/10.1016/j.jcm.2016.02.012

25. Tavakol M, Dennick R. Making sense of Cronbach's alpha. Int J Med Educ. 2011;2:53-55. https://doi.org/10.5116/ijme.4dfb.8dfd

26. Goss DA. Ocular accommodation, convergence, and fixation disparity: A manual of clinical analysis. 2nd ed. Boston, MA: Butterworth-Heinemann; 1995.

27. Scheiman $M$, Wick B. Clinical management of binocular vision: Heterophoria, accommodative, and eye movement disorders. 2nd ed. Philadelphia, PA Lippincott Williams \& Wilkins; 2008.
28. Matheron E, Kapoula Z. Vertical phoria and postural control in upright stance in healthy young subjects. Clin Neurophysiol. 2008;119(100):2314-2320. https:// doi.org/10.1016/j.clinph.2008.06.016

29. Jackson DN, Bedell HE. Vertical heterophoria and susceptibility of visually-induced motion sickness. Strabismus. 2012;20(1):17-23. https://doi.org/10.3109/092739 72.2011 .650813

30. Amos JF, Rutstein RP. Vertical deviations. In: Amos JF, editor. Diagnosis and management in vision care. Boston, MA: Butterworth-Heinemann, 1987; p. 515-583.

31. Benjamin WJ. Borish's clinical refraction. 2nd ed. St. Louis, MO: ButterworthHeinemann, 2006; p. 1020-1024.

32. Rosner AJ, Feinberg DL. Vertical Heterophoria: A common cause of dizziness and headache. Otolaryngol Head Neck Surg. 2005;133(2):P41-P42. https://doi. org/10.1016/j.otohns.2005.05.073 